\title{
Neural representation of reward in recovered depressed patients
}

\author{
Ciara McCabe $\cdot$ Philip J. Cowen $\cdot$ Catherine J. Harmer
}

Received: 2 February 2009 /Accepted: 13 May 2009 /Published online: 16 June 2009

C) The Author(s) 2009. This article is published with open access at Springerlink.com

\begin{abstract}
Introduction Anhedonia, a loss of interest and pleasure in normally rewarding stimuli, is a key diagnostic criterion for major depression. It has been suggested that deficits in the processing of reward-relevant stimuli could represent an endophenotype for depression. We hypothesized that people at risk of depression by virtue of a personal history of the illness would show impaired neural responses to a primary rewarding stimulus.

Materials and methods Using functional magnetic resonance imaging, we measured the neural response to the sight and flavor of chocolate, and their combination, in 13 unmedicated recovered patients with a history of major depression and 14 healthy controls matched for age and gender. We also examined a control aversive condition consisting of the sight of moldy strawberries and a corresponding unpleasant taste. Participants simultaneously recorded subjective ratings of "pleasantness," "intensity," and "wanting."

Results and discussion Despite no differences between the groups in stimulus ratings, patients showed decreased neural responses to the pleasant stimulus in the ventral striatum and increases in the caudate nucleus to the aversive stimulus. Furthermore, patients had a diminished neural supralinearity response (the potentiation produced by simultaneous presentation of the sight and flavor of the stimuli) in the prefrontal cortex for both aversive and pleasant conditions. Patients recovered from depression appear to have deficits in the neural basis of reward and
\end{abstract}

C. McCabe $(\bowtie) \cdot$ P. J. Cowen $\cdot$ C. J. Harmer

University Department of Psychiatry,

Warneford Hospital, University of Oxford,

Oxford, UK

e-mail: ciara.mccabe@psych.ox.ac.uk may also have impairments in the cross-modal integration of sensory stimuli.

Conclusion These findings support the view that abnormal neural responses to reward may be an endophenotype for depression and a potential target for intervention and prevention strategies.

Keywords fMRI · Depression - Reward C Chocolate · Endophenotype

\section{Introduction}

Anhedonia, or loss of interest and pleasure in activities customarily enjoyed, is a key diagnostic criterion for depressive disorder in both major psychiatric diagnostic systems: the Diagnostic Statistical Manual (DSM-IV) and the International Statistical Classification of Diseases (ICD). Anhedonia is an important symptom because, although pervasive negative affect is a feature of most emotional disorders, anhedonia is quite specific for depression (Dryman and Eaton 1991). In addition, the presence of anhedonia in depressed patients could have important implications for pathophysiology because it is suggestive of changes in the neurobiological mechanisms that underpin motivation and reward (Nestler and Carlezon 2006).

Anhedonia is usually regarded as a symptom of acute depression which resolves with clinical recovery. However, anhedonia also has some trait characteristics and has been suggested as a potential endophenotypic marker of major depression (Hasler et al. 2004). For example, an epidemiological study showed that the presence of anhedonic features was a strong predictor of the onset of major depression over the following year (Dryman and Eaton 1991). In a group of patients with chronic depression followed up for a year, 
Schrader (1997) found that anhedonic symptoms remained fairly constant despite a substantial remission in severity of overall depression. In addition, in the same patients, anhedonia scores correlated with the presence of depression in first-degree relatives, suggesting a genetic link between anhedonia and the risk of depression.

Brain imaging studies such as functional magnetic resonance imaging (fMRI) have made substantial progress in identifying the neural systems underlying reward processes in humans. Thus, monetary reward (O'Doherty et al. 2001a), pleasurable responses to music (Blood and Zatorre 2001), and the viewing of pleasant pictures (Lane et al. 1997) have all been associated with increased neural activity within the ventral striatum and ventromedial prefrontal cortex (VMPFC). This is consistent with studies in animals indicating that dopaminergic activity in the ventral striatum is important in mediating positive reinforcing effects of rewards such as food and sex and may play a key role in the incentive motivation and anticipation of these appetitive stimuli. By contrast, the VMPFC appears to integrate sensory experiences from different modalities and contribute to the subjective experiences of reinforcing stimuli (Robbins and Everitt 1996; Cardinal et al. 2002).

Some studies have investigated the neural representation of reward in acutely depressed patients. While the reward stimuli employed have generally been indirect, for example, happy facial expressions or positively valenced words, studies have found abnormalities in the neural circuitry supporting reward mechanisms. For example, reduced ventral striatal responses have been reported to positively valenced words in depression (Epstein et al. 2006), while Knutson et al. (2008) found an altered pattern of responses in the anterior cingulate cortex to monetary reward. A similar network was identified in a study examining behavioral and neural response to feedback information during a gambling task where depressed patients showed decreased responses in the ventral striatum and anterior cingulate to feedback information of "winning" or "losing" money and did not adjust their response times accordingly unlike the control group (Steele et al. 2007). However, it is unknown whether neural differences in responses to reward are seen during remission from depression and may form a trait vulnerability marker for this disorder.

The aim of the present study was to assess the neural substrate of reward in unmedicated and clinically recovered depressed patients using a paradigm involving the sight and taste of chocolate as a direct reward and an aversive taste and picture condition as a control. This design allows us to examine whether a history of depression affects responses to all tastes and affective pictures or whether there is a specific blunting of response to positive stimuli. The use of similar paradigms, in conjunction with functional neuroimaging, has permitted a clear delineation of the neural systems support- ing the representation of reward in humans (Rolls and McCabe 2007). We predicted that recovered depressed patients would have impaired neural responses to the chocolate reward within the anterior cingulate, the VMPFC, and the ventral striatum in which we and other authors have found activations in previous studies to unconditioned reward stimuli (Rolls and McCabe 2007; O'Doherty et al. 2001b; McCabe and Rolls 2007; Rolls et al. 2003). By contrast, we expected the neural response to the aversive taste and sight condition to be enhanced in the recovered depressed patients in neural circuitry mediating disgust and aversive processing including the amygdala, caudate, and anterior insula (Fitzgerald et al. 2004; Zald et al. 2002).

\section{Materials and methods}

\section{Participants}

Fourteen healthy control volunteers (five men, nine women) and 13 recovered depressed patients (three men, 11 women) were recruited for this study. Ethical approval was provided by the Central Oxford Research Ethics Committee and written informed consent was obtained from all participants before screening and after the complete description of the study was given. Exclusion criteria for all subjects consisted of current or past history of alcohol or drug dependency, pregnancy, and any contraindications to MRI, e.g., pacemaker, mechanical heart valve, hip replacement, metal implants. Furthermore, the control group were determined to be free of current or past axis 1 disorder (including anxiety disorders, depression, eating disorder, psychosis, and substance abuse) on the structured clinical interview for DSM-IV (Spitzer et al. 2004). To be included in the recovered depressed group, the participants were required to meet the criteria for at least one episode of major depression as a primary diagnosis and also to be free of current or past comorbid axis 1 disorder (including anxiety disorders, eating disorders, psychosis, and substance abuse) on the structured clinical interview for DSM-IV (Spitzer et al. 2004). Recovery was determined in the same week as the study through clinical interview and with the Hamilton Depression Scale (HAM-D; Hamilton 1960) using a cut-off score of 8 . None of the participants took current medication apart from the contraceptive pill and the recovered depressed group had been free of antidepressant medication for a mean of 4.9 years (range $=$ 1.7-7 years). Four people in the recovered depressed group had received citalopram for 6 months and one person had citalopram for 1 year. One person in the recovered depressed group received 6 months treatment with fluoxetine and the other seven recovered depressed participants were treatment naïve. 
All subjects were rated on the following questionnaires: Beck Depression Inventory (BDI; Beck et al. 1961), the Fawcett-Clarke Pleasure Scale (FCPS; Fawcett et al. 1983), and the Snaith-Hamilton Pleasure Scale (SHAPS; Snaith et al. 1995) approximately 1 week before scanning. The participants also completed a "chocolate questionnaire" to measure liking, craving, and frequency of eating chocolate (Rolls and McCabe 2007) and the body mass index (BMI) for each individual was also calculated.

\section{Overall design}

We compared brain responses to reward-related and aversive stimuli in a group of unmedicated recovered depressed volunteers and healthy controls. Each of the following conditions were applied nine times in a randomized order (see Table 1): chocolate in the mouth, chocolate picture, chocolate in the mouth with chocolate picture, strawberry in the mouth, strawberry picture, and strawberry in the mouth with strawberry picture. Examining the responses to each stimulus alone and in combination allowed a supralinearity analysis to be performed to identify those neural circuits which showed a potentiated response with cross-modal presentation of stimuli (e.g., those areas showing greater activation to the combination of the sight and flavor of chocolate than to the sum of the activations produced by the sight alone and by the flavor alone of chocolate). Subjective effects of each stimulus were measured by psychophysical ratings of pleasantness, inten-

Table 1 Stimuli

A list of the stimulus conditions

\begin{tabular}{|c|c|}
\hline Condition 1 & Chocolate in the mouth + gray visual stimulus \\
\hline Condition 2 & Picture of chocolate \\
\hline Condition 3 & Chocolate in the mouth + a picture of chocolate \\
\hline Condition 4 & Strawberry in the mouth + gray visual stimulus \\
\hline Condition 5 & Picture of moldy strawberries \\
\hline Condition 6 & $\begin{array}{l}\text { Strawberry in the mouth }+ \text { a picture of moldy } \\
\text { strawberries }\end{array}$ \\
\hline Rinse condition & $\begin{array}{l}\text { Tasteless rinse control solution }+ \text { gray visual } \\
\text { stimulus }\end{array}$ \\
\hline Supralinearity choc & Condition 3 - Condition 1 - Condition 2 \\
\hline Supralinearity straw & Condition 6 - Condition 4 - Condition 5 \\
\hline
\end{tabular}

The term "chocolate in the mouth" refers to the intraoral delivery through a Teflon tube of $0.5 \mathrm{~mL}$ of a fine liquid chocolate, which was identical for all such trials and which could not be seen by the subject. The term "strawberry in the mouth" refers to the intraoral delivery of $0.5 \mathrm{~mL}$ of an unpleasant strawberry flavored drink through a tube that was also could not be seen by the subject. The term "picture of chocolate" refers to a picture of a bar of brown, i.e., milk, chocolate shown on the display screen and the term "picture of moldy strawberries" refers to a picture of moldy strawberries sity, and wanting for the stimuli made on every trial by the subjects during the fMRI acquisition. The participants were instructed not to eat chocolate for $24 \mathrm{~h}$ before the scan and to eat only a small lunch on the day of scanning. Scanning took place at 2.30-4P.M. Mood state was recorded on the study day with the BDI.

\section{Stimuli}

Stimuli were delivered to the subject's mouth through three Teflon tubes (one for the tasteless rinse control described below, one for chocolate taste, and one for strawberry taste) that were held between the lips. Each Teflon tube of approximately $3 \mathrm{~m}$ in length was connected to a separate reservoir via a syringe and a one-way syringe activated check valve (Model 14044-5, World Precision Instruments), which allowed $0.5 \mathrm{~mL}$ of any stimulus to be delivered manually at the time indicated by the computer. The chocolate was formulated to be liquid at room temperature, with a list of the six stimulus conditions described above in Table 1. A control tasteless solution (distilled $\mathrm{H}_{2} \mathrm{O}$ ) was used as a rinse between trials (Table 1), and when subtracted from the effects of the other stimuli, allowed somatosensory and any mouth movement effects to be subtracted from the effects produced by the other oral stimuli. This allows the taste, texture, and olfactory areas to be shown independently of any somatosensory effects produced by introducing a fluid into the mouth (O'Doherty et al. 2001b). The aversive stimulus was a strawberry drink (Rosemount Pharmaceuticals) which was reported to be unpleasant by pilot screenings with volunteer tasters. Both the liquid chocolate and the strawberry had approximately the same sweetness and texture which enabled them to pass freely through the Teflon delivery tubes.

\section{Experimental procedure}

At the beginning of each trial, one of the six stimuli chosen by random permutation was presented. If the trial involved an oral stimulus, this was delivered in a $0.5-\mathrm{mL}$ aliquot to the subject's mouth. At the same time, at the start of the trial, a visual stimulus was presented, which was either the picture of chocolate, of moldy strawberries, or a gray control image of approximately the same intensity. The image was turned off after $7 \mathrm{~s}$ at which time a small green cross appeared on a visual display to indicate to the subject to swallow what was in the mouth. After a delay of $2 \mathrm{~s}$, the subject was asked to rate each of the stimuli for pleasantness on that trial (with +2 being very pleasant and -2 very unpleasant), for intensity on that trial $(0$ to +4$)$, and for current wanting for chocolate $(+2$ for wanting chocolate very much, 0 for neutral, and -2 for very much not wanting chocolate). The ratings were made with a visual analog rating scale in which the subject moved 
the bar to the appropriate point on the scale using a button box. Each rating period was $5 \mathrm{~s}$ long. After the last rating, the gray visual stimulus indicated the delivery of the tasteless control solution that was also used as a rinse between stimuli, and this was administered in exactly the same way as a test stimulus and the subject was cued to swallow after $7 \mathrm{~s}$ by the green cross. The tasteless control was always accompanied by the gray visual stimulus. On trials on which only the picture of chocolate was shown, there was no rinse but the gray visual stimulus was shown in order to allow an appropriate contrast as described below. There was then a 2-s delay period similar to other trials that allowed for swallowing followed by a 1-s gap until the start of the next trial. A trial was repeated for each of the six stimulus conditions shown in Table 1, and the whole cycle was repeated nine times. The instruction given to the subject was (on oral delivery trials) to move the tongue once as soon as a stimulus or tasteless solution was delivered (at the time when the gray visual stimulus was turned on) in order to distribute the solution round the mouth to activate the receptors for taste and smell and then to keep still for the remainder of the 7-s period until the green cross was shown, when the subject could swallow. This procedure has been shown to allow taste effects to be demonstrated clearly with fMRI, using the procedure of subtracting any activation produced by the tasteless control from those produced by a taste or other stimulus (O'Doherty et al. 2001b).

\section{fMRI scan}

The experimental protocol consisted of an event-related interleaved design using in random permuted sequence the six stimuli described above and shown in Table 1. Images were acquired with a 3.0-T VARIAN/SIEMENS wholebody scanner at the Centre for Functional Magnetic Resonance Imaging at Oxford (FMRIB) where T2*weighted EPI slices were acquired every $2 \mathrm{~s}(\mathrm{TR}=2)$. Imaging parameters were selected to minimize susceptibility and distortion artifact in the orbitofrontal cortex (Wilson et al. 2002). Coronal slices (25) with in-plane resolution of $3 \times 3 \mathrm{~mm}$ and between-plane spacing of $4 \mathrm{~mm}$ were obtained. The matrix size was $64 \times 64$ and the field of view was $192 \times 192 \mathrm{~mm}$. Acquisition was carried out during the task performance, yielding 972 volumes in total. A wholebrain T2*-weighted EPI volume of the above dimensions and an anatomical T1 volume with coronal plane slice thickness of $3 \mathrm{~mm}$ and in-plane resolution of $1.0 \times 1.0 \mathrm{~mm}$ were also acquired.

\section{fMRI analysis}

The imaging data were analyzed using SPM5 (http://www. fil.ion.ucl.ac.uk/spm/). Preprocessing of the data used
SPM5 realignment, reslicing with sinc interpolation, normalization to the Montreal Neurological Institute (MNI) coordinate system and spatial smoothing with a 6-mm full width at half maximum isotropic Gaussian kernel and global scaling (Collins et al. 1994). The time series at each voxel were low-pass filtered with a hemodynamic response kernel. Time series nonsphericity at each voxel was estimated and corrected for (Friston et al. 2002), and a high-pass filter with a cut-off period of $128 \mathrm{~s}$ was applied. In the single event design, a general linear model was then applied to the time course of activation where stimulus onsets were modeled as single impulse response functions and then convolved with the canonical hemodynamic response function (Friston et al. 1994). Linear contrasts were defined to test specific effects. Time derivatives were included in the basic functions set. Following smoothness estimation (Kiebel et al. 1999), linear contrasts of parameter estimates were defined to test the specific effects of each condition with each individual dataset. Voxel values for each contrast resulted in a statistical parametric map of the corresponding $t$ statistic, which was then transformed into the unit normal distribution (SPM Z). The statistical parametric maps from each individual dataset were then entered into second-level, random effects analyses accounting for both scan-to-scan and subject-to-subject variability. More precisely, the sets of individual statistical maps corresponding to a specific effect of interest were entered as covariates in multiple regression models as implemented in SPM5, and the corresponding group effects were assessed by applying linear contrasts (again following smoothness estimation) to the (second-level) parameter estimates generating a $t$ statistics map for each group effect of interest. SPM converts the $t$ statistics to $Z$ scores (Table 4). Reported $p$ values for each cluster based on this group analysis are fully corrected for the number of comparisons (resels) in the entire brain volume ("wholebrain" multiple comparisons) (Worsley et al. 1996) for which $p<0.05$ FWE. We report small volume corrections for brain regions in which we had an a priori hypothesis (described in the "Introduction" section) as follows: ventral striatum [-4 16 -12] and pregenual cingulate cortex [4 30 8] (Rolls and McCabe 2007; McCabe et al. 2008), medial prefrontal cortex $\left[\begin{array}{lll}0 & 54 & -12\end{array}\right]$ and lateral orbitofrontal cortex [-16 28 -18] (Rolls et al. 2003), caudate nucleus [-8 18 10] (Fitzgerald et al. 2004), and amygdala [-18 -10 -14] (Zald et al. 2002). Peaks within $10 \mathrm{~mm}$ of these and which had a $p$ value of at least $<0.001$ uncorrected in the whole-brain analysis and with a cluster threshold of 30 contiguous voxels $(k=30)$ had applied small volume (FDR) corrections for multiple comparisons (Worsley et al. 1996) with a radius corresponding to the full width at half maximum of the spatial smoothing filter used. The percent change in the blood oxygen level-dependent (BOLD) signal was extracted 
for the different conditions for illustration from the peak voxel within the regions of significant group difference identified from the contrast analyses using SPM5 plots of contrast estimates and signal effect sizes. For illustration purposes only, WFU Pick Atlas (Maldjian et al. 2003) was used to display activations (http://www.fmri.wfubmc.edu/ $\mathrm{cms}$ /software). Coordinates of the activations are listed in the stereotactic space of the MNI's ICBM152 brain (Table 4).

\section{Results}

Demographic details and mood ratings

The two groups were matched for age, gender, BMI, and chocolate liking (Table 2). There were no differences between the control group and the recovered depressed group in the measures of anhedonia (SHAPS, FCPS) and the HAM-D. However, the recovered depressed group scored significantly higher on the BDI (Table 2).

\section{Ratings of stimuli}

Ratings of pleasantness, intensity, and wanting for the stimuli were obtained during the scanning on each trial for every condition for the healthy controls and the recovered depressed subjects. All subjects rated the strawberry picture and taste as unpleasant and the chocolate stimuli as pleasant. Using a repeated-measures analysis of variance for the pleasantness, intensity, and wanting ratings, there were no significant differences between the two groups and their ratings of pleasantness over the six stimuli $[F(1,25)=$ $0.01, p=0.9]$, intensity over the six stimuli $[F(1,25)=0.68$,

Table 2 Group demographic and psychosocial measures

\begin{tabular}{lll}
\hline Measure & $\begin{array}{l}\text { Recovered depressed } \\
(n=13), \text { mean (SD) }\end{array}$ & $\begin{array}{l}\text { Controls } \\
(n=14), \\
\text { mean (SD) }\end{array}$ \\
\hline Age (years) & $27.8(6.6)$ & $28.5(6.3)$ \\
Gender (male) & $3 / 13$ & $5 / 14$ \\
BDI & $5.5(6.3)$ & $0.8(1.3)^{*}$ \\
HAM-D & $2.3(2.9)$ & $0.5(1.1)$ \\
FCPS & $118(33.7)$ & $118(33.5)$ \\
SHAPS & $23(6)$ & $19.25(6)$ \\
BMI & $22.1(2.5)$ & $22.2(5.14)$ \\
Choc craving & $6.1(2.4)$ & $6.8(2.1)$ \\
Choc liking & $7.9(1.7)$ & $8.0(1.7)$ \\
Choc freq eat & $5.0(3.2)$ & $5.0(3.1)$ \\
\hline
\end{tabular}

$B D I$ Beck Depression Inventory, HAM-D Hamilton Depression Score, FCPS Fawcett-Clarke Pleasure Scale, SHAPS Snaith-Hamilton Pleasure Scale, $B M I$ body mass index

${ }^{*} p=0.01$ (all other $p>0.1$ ), independent samples $t$ tests $p=0.41]$, or wanting over the six stimuli $[F(1,25)=0.126$, $p=0.79$ ] (see Table 3 ).

\section{fMRI responses}

Table 4 provides a summary of the results for each contrast, first across all subjects to indicate the main effect of task and then with the interaction with group (recovered depressed vs. control). The fMRI results remained significant when the BDI scores were added as a covariate.

\section{Main effect of task}

As expected, the taste stimuli of chocolate and strawberry activated an overlapping region of the anterior insula, i.e., the primary taste cortex, in both the controls and the recovered depressed subjects. The rewarding stimuli chocolate taste and chocolate picture activated reward-relevant circuitry including the ventral striatum, the cingulate cortex, and the VMPFC extending into the medial orbitofrontal cortex. By contrast, the unpleasant stimuli of strawberry taste and sight of the moldy strawberries activated areas involved in aversive processing including the amygdala and a more lateral posterior part of the insula cortex (distinct from that activated by the tastes alone).

\section{Effects of group}

As expected, there was no significant difference in response to the taste stimuli between the two groups in the primary taste cortex, confirming that the sensory experience of these stimuli was associated with a similar neural response across groups (Fig. 1a, b).

Chocolate reward: sight and taste

The recovered depressed group showed reduced responses to the sight and taste of chocolate in areas known to play a key role in reward, including the ventral striatum and cingulate cortex (Fig. 2a). There were no areas where the recovered depressed patients showed increased responses relative to the controls for the chocolate reward condition (Table 4).

Strawberry: sight and taste

Contrary to the reduced BOLD responses seen to the chocolate reward, the unpleasant strawberry picture increased activation in the recovered depressed group compared to the control group in the bilateral caudate, as illustrated in Fig. 3a, b. To ensure the results validity and that the activations were not in the ventricles, the peak activations were overlaid on an average individual subject 
Table 3 Subjective ratings

\begin{tabular}{|c|c|c|c|c|c|c|}
\hline \multirow[t]{2}{*}{ Condition } & \multirow{2}{*}{$\begin{array}{l}\begin{array}{l}\text { Recovered } \\
\text { depressed }\end{array} \\
\text { Pleasantness, } \\
\text { mean (SD) }\end{array}$} & \multirow[t]{2}{*}{ Controls } & \multirow{2}{*}{$\begin{array}{l}\begin{array}{l}\text { Recovered } \\
\text { depressed }\end{array} \\
\text { Intensity, } \\
\text { mean (SD) }\end{array}$} & \multirow[t]{2}{*}{ Controls } & \multirow{2}{*}{$\begin{array}{l}\begin{array}{l}\text { Recovered } \\
\text { depressed }\end{array} \\
\text { Wanting, } \\
\text { mean (SD) }\end{array}$} & \multirow[t]{2}{*}{ Controls } \\
\hline & & & & & & \\
\hline Chocolate in the mouth + gray visual stimulus & $1.09(0.15)$ & $1.16(0.28)$ & $1.47(0.42)$ & $1.83(0.5)$ & $1.05(0.21)$ & $1.24(0.3)$ \\
\hline Picture of chocolate & $1.14(0.28)$ & $1.02(0.36)$ & $1.38(0.7)$ & $1.36(0.58)$ & $1.05(0.35)$ & $1.11(0.4)$ \\
\hline Chocolate in the mouth + a picture of chocolate & $1.29(0.23)$ & $1.4(0.26)$ & $1.76(0.29)$ & $2.18(0.62)$ & $1.27(0.27)$ & $1.38(0.29)$ \\
\hline Strawberry in the mouth + gray visual stimulus & $-0.85(0.93)$ & $-0.75(0.95)$ & $1.91(0.56)$ & $1.97(0.76)$ & $-0.91(0.98)$ & $-0.97(0.87)$ \\
\hline Picture of moldy strawberries & $-1.02(0.42)$ & $-1.15(0.47)$ & $1.34(0.69)$ & $1.41(0.84)$ & $-1.35(0.35)$ & $-1.33(0.46)$ \\
\hline Strawberry in the mouth + a picture of moldy strawberries & $-1.14(0.7)$ & $-1.22(0.51)$ & $2(0.68)$ & $2.04(0.75)$ & $-1.2(0.77)$ & $-1.31(0.34)$ \\
\hline
\end{tabular}

T1 template. Furthermore, we investigated the individual subjects' activations to this condition across groups to ensure valid registrations and activations within the cortex. No other comparisons were statistically significant between groups.

Supralinearity: the sight and flavor of chocolate compared to the sum of the activations to the sight alone and the flavor alone

In our previous studies in healthy control subjects, we showed that combinations of pictures and tastes induced greater activations in reward areas of the brain than when the pictures or the tastes were presented alone (Rolls and McCabe 2007; McCabe and Rolls 2007). Therefore, in this experiment, we compared activations to a combination of the sight and flavor of chocolate with the sum of the activations to the sight alone and the flavor alone to assess any differences in cross-modal integration of reward between patients and controls. We found greater supralinearity in the VMPFC extending into the medial orbitofrontal cortex in the controls compared to the recovered depressed group, as illustrated in Fig. 4a, b. These findings show that the increased activation due to the combination of the flavor of chocolate in the mouth with the sight of chocolate is less in recovered depressed patients than in controls.

Supralinearity: the sight and flavor of strawberry compared to the sum of the activations to the sight alone and the flavor alone

To investigate whether the groups differed in the crossmodal integration of aversive stimuli, a supralinearity analysis was performed comparing activations to a combination of the sight and flavor of strawberry with the sum of the activations to the sight alone and the flavor alone. We found greater supralinearity in the control subjects in the bilateral lateral orbitofrontal cortex relative to the recovered depressed group (Fig. 4c, d). This appeared to be driven by both decreased responses to the stimuli combined and increased responses to the picture stimulus presented alone.

\section{Discussion}

The main finding of our study is that unmedicated recovered depressed patients demonstrate abnormalities in the neural representation of reward to the sight and taste of chocolate. Judged by the visual analog scale ratings, these impaired neural responses were not apparently attributable to subjective changes in how the reward was anticipated or experienced. Moreover, in keeping with their recovered status, the patients did not suffer from symptomatic anhedonia as captured by standardized ratings scales such as the FCPS and the SHAPS. Importantly, the neural representations of the taste of chocolate and strawberry, judged by activations in the insula taste cortex, were not significantly different in recovered patients and controls; this suggests that the sensory qualities of the taste stimuli did not differ between the two groups. Our findings are, therefore, consistent with the proposal that deficiencies in the neural basis of reward mechanisms may be a trait abnormality in people at risk of depression.

The ventral striatum plays a key role in reward processes and is believed to be involved in the anticipation of reward and more specifically in learning to predict rewards, thereby biasing behaviors in a reward-seeking direction (Robbins and Everitt 1996; Hare et al. 2008). Furthermore, studies in acutely depressed patients using secondary rewards such as positive words or monetary reward have shown impaired neural responses in ventral striatum responses (Epstein et al. 2006) and reduced BOLD responses in this brain area correlate specifically with symptomatic anhedonia in medicated patients (Keedwell et al. 2005). Using behavioral and neural response to feedback 
Table 4 Regions showing main effect of task and interaction with group (controls vs. recovered depressed) rec dep recovered depressed, cing cingulate cortex, $s$ superior, $m O F C$ medial orbitofrontal cortex, $l O F C$ lateral orbitofrontal cortex

${ }^{*} p<0.05$, whole-brain corrected (FWE), FDR small volume correction; $* * p=0.06$, after adding the BDI as a covariate of no interest
Brain area

$x$

$y$

$z$

$Z$ score

$p$ value

MNI coordinates

Chocolate in the mouth (all subjects)

Anterior insula

$\begin{array}{rrrll}34 & 18 & 0 & 5.3 & <0.001 \\ -34 & 14 & 4 & 4.94 & <0.003 \\ 10 & 10 & 0 & 4.31 & <0.001 \\ -10 & 6 & -2 & 4.34 & <0.001\end{array}$

Chocolate in the mouth (controls vs. rec dep)

Ventral striatum

Pregenual cing

Subgenual cing

Sight of chocolate (all subjects)

Striatum

Cingulate cortex

Pregenual cing

Orbito/insula trans area

Thalamus

$\begin{array}{rrrll}-6 & -2 & -6 & 3.13 & 0.03 * * \\ -6 & 34 & 2 & 3.3 & 0.02 * \\ 10 & 38 & -20 & 3.08 & 0.03 *\end{array}$

$\begin{array}{lllll}-10 & 2 & 2 & 5.6 & <0.001\end{array}$

$\begin{array}{lllll}16 & 2 & 2 & 5.49 & <0.001\end{array}$

$\begin{array}{lllll}-6 & 2 & 46 & 6.5 & <0.001\end{array}$

$\begin{array}{lllll}-4 & 30 & 2 & 5.52 & <0.001\end{array}$

$\begin{array}{lllll}44 & 14 & -2 & 6.5 & <0.001\end{array}$

$\begin{array}{lllll}-34 & 24 & 0 & 5.82 & <0.001\end{array}$

$\begin{array}{lllll}-10 & -18 & -8 & 6.35 & <0.001\end{array}$

$\begin{array}{lllll}12 & -22 & -10 & 6.2 & <0.001\end{array}$

Chocolate in the mouth with the sight of chocolate (controls vs. rec dep)

Ventral striatum

Caudate

Anterior cing

Pregenual cing

Posterior cing

S temporal gyrus

Supralinearity: chocolate taste and sight of chocolate minus chocolate taste alone and chocolate picture alone (controls vs. rec dep)

$\mathrm{VMPFC} / \mathrm{mOFC}$

Strawberry in the mouth (all subjects)

Anterior insula

Sight of moldy strawberries (all subjects)

Insula

Putamen

Thalamus

Amygdala

Supralinearity: strawberry taste and sight of moldy strawberries minus the strawberry taste alone and the strawberry picture alone (controls

vs. rec dep)

1OFC

Region of increased activation in the recovered depressed group compared to the control group Sight of moldy strawberries (rec dep vs. controls)

Caudate

\begin{tabular}{rrrrl}
12 & 10 & 22 & 3.5 & $0.004^{*}$ \\
-8 & 8 & 16 & 3.2 & $0.01 *$ \\
\hline
\end{tabular}

$0.01^{*}$

$<0.03$

$<0.03$

$<0.02$

$<0.02$

$<0.001$

$<0.03$

$0.03 *$

$<0.001$

$<0.001$

$<0.001$

$<0.001$

$<0.001$

$<0.001$

$<0.01$

$0.01 *$ 
Fig. 1 The effects of taste in the mouth, across all subjects for taste stimuli. a Axial, coronal, and sagittal images depicting activation in the primary taste cortex in the anterior insula. b The \% BOLD signal change in the anterior insula for the recovered depressed and control groups, no significant group difference a

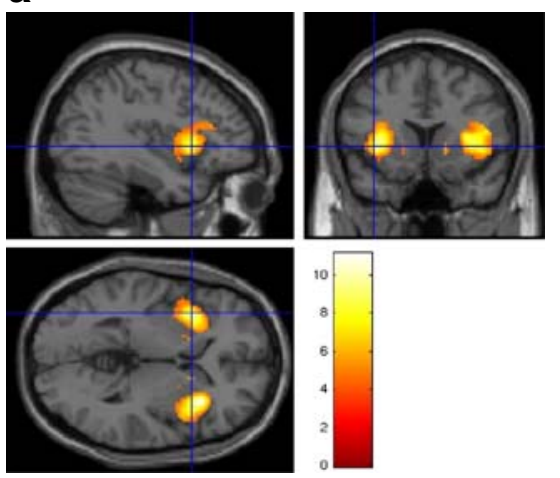

b

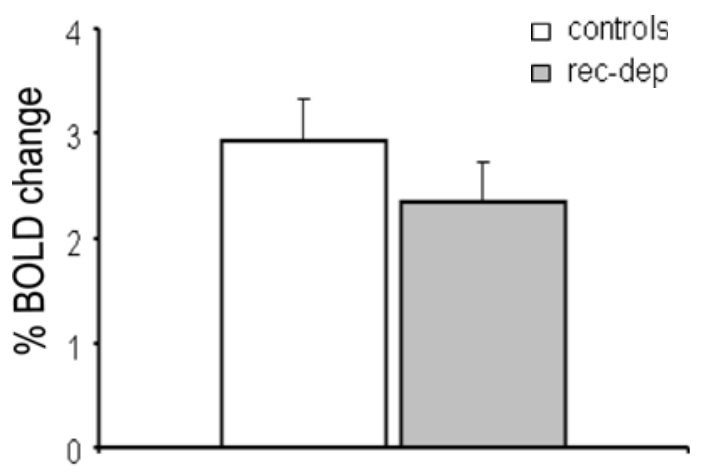

information during a gambling task, a recent study has shown that patients compared to controls have decreased activations in the ventral striatum to the positive reinforcement of "wins" and that they failed to adjust their response times to the positive feedback and that this correlated with measures of anhedonia (Steele et al. 2007). Our findings indicate that abnormalities in ventral striatal response to primary reward experience can also be seen in unmedicated patients who have recovered from depression and show no differences in subjective experience of the reward stimulus. Such results suggest that impaired ventral striatal activity may, at least in part, be independent of symptomatic state and could form part of the vulnerability to experience future depressive episodes. For example, McClure et al. (2004) proposed that impaired activity in the ventral striatum to reward could be associated with a lessened ability to learn stimulus-reward associations resulting in a failure to bias goal-directed behavior appropriately. Accordingly, trait deficits of this sort could result in difficulties in making complex social decisions leading to the consequent selection of potentially adverse environments. Individuals thus affected might be expected to run a greater risk of experiencing clinical mood disorders. Such a pattern is consistent with the hypothesis that deficits in the neural basis of reward anticipation or the reward experience itself may be an endophenotype for depression. Since the current sample were predominantly female, however, it will be important to confirm whether similar effects are seen in male recovered depressed patients or whether there are any sex differences in these responses.

The VMPFC is involved in the integration of rewardrelevant information from different sensory modalities and in mediating the subjective hedonic experiences of these stimuli (Robbins and Everitt 1996; Cardinal et al. 2002). Consistent with this, the supralinearity analysis revealed a diminished response in recovered depressed patients in the VMPFC extending down into the medial part of the orbitofrontal cortex. This supralinearity response is believed to represent cross-modal sensory potentiation where combined visual and taste stimuli produce a greater combined effect on reward than the individual sensory components added together, consistent with real-world reward experiences (i.e., seeing and tasting food together). This part of the brain has been implicated specifically in the evaluation of positive rewards, so the impaired response in recovered depressed patients may be consistent with a further attenuation of neural reward mechanisms in a key cortical region believed to contribute to hedonic state. Such a

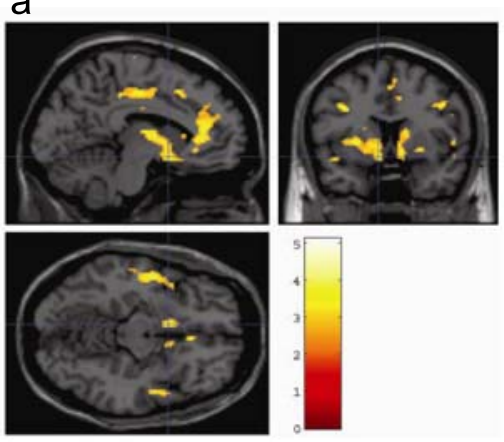

b

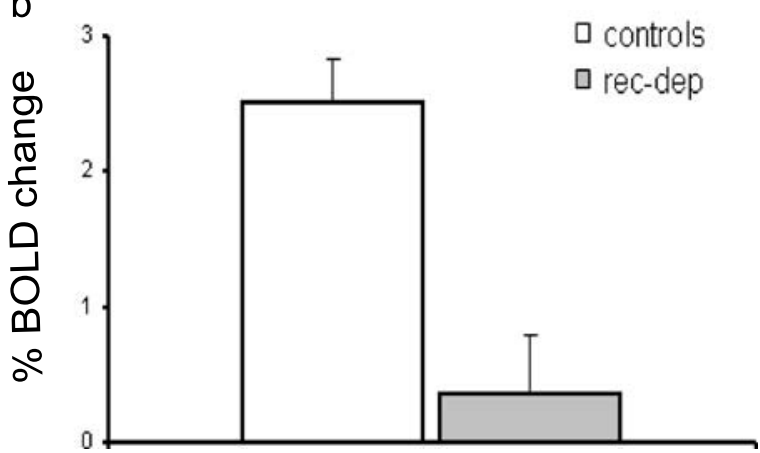

cingulate cortex. b The \% BOLD signal change in the ventral striatum for the recovered depressed and control groups ( $\left[\begin{array}{lll}-8 & 8 & -12\end{array}\right], p<0.03$, fully corrected)
Fig. 2 Chocolate in the mouth with the sight of chocolate condition: controls vs. recovered depressed. a Axial, coronal, and sagittal images depicting significantly decreased activations in the recovered depressed group in bilateral ventral striatum, caudate, pregenual, and anterior 
Fig. 3 Sight of moldy strawberries: recovered depressed vs. controls. a Axial, coronal, and sagittal images depicting significantly increased activations in the recovered depressed group in the bilateral caudate. b The \% BOLD signal change in the caudate for the recovered depressed and control groups ([12 1022$], p<0.004$, small volume correction) a
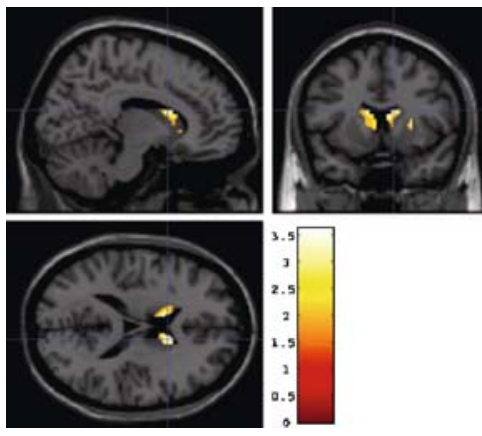

b

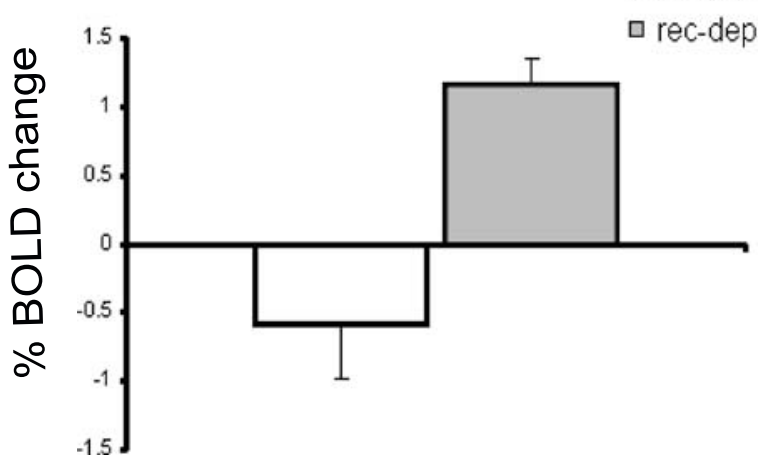

impairments may arise either as direct deficits in the function of the medial prefrontal cortex or through poor communication and integration of individual sensory inputs.

We also assessed the response of patients and controls to an aversive sight and taste condition to determine whether any decrease in neural responses to the reward of chocolate might represent a general blunting of neural responses to motivational stimuli in general, whether positive or negative. Both patients and controls rated the strawberry taste and picture as equally aversive and we found no blunting of neural response in the recovered depressed subjects to either stimulus considered separately. However, once again, the supralinearity analysis revealed a blunting of BOLD response in the recovered depressed patients relative to controls, this time in the lateral orbitofrontal cortex. The extraction of the signal change for this comparison, however, revealed that this may be partly driven by exaggerated neural response in this area when the aversive picture stimulus was presented alone but not in combination with the taste.

The lateral orbitofrontal cortex is believed to be particularly involved in the representation of unpleasant stimuli (Rolls et al. 2003; Elliott et al. 2000; Kringelbach and Rolls 2004) and in the selection of actions that override automatic and motivationally (reward) driven response tendencies (Elliott et al. 2000; Passingham et al. 2000; Rushworth et al. 2007). This suggests that depressed patients may also have impairments in the cortical representations of aversive stimuli. Clinically, for example, it has long been known that some depressed patients experience a rather general loss of emotional response; for
Fig. 4 Supralinearity condition for chocolate and strawberry conditions: controls vs. recovered depressed. a Axial, coronal, and sagittal images depicting significantly decreased activations in the recovered depressed group in the VMPFC/medial orbitofrontal cortex. b The \% BOLD signal change for the chocolate picture plus chocolate taste condition minus the chocolate picture alone minus the chocolate taste alone in the VMPFC/medial orbitofrontal cortex ([8 56 -12], $p=0.03$, small volume correction). c Axial, coronal, and sagittal images depicting significantly decreased activations in the recovered depressed group in the lateral orbitofrontal cortices. d The \% BOLD signal change for the strawberry picture plus strawberry taste condition minus the strawberry picture alone minus the strawberry taste alone in the lateral orbitofrontal cortex ([20 $22-8$ ], $p=0.01$, small volume
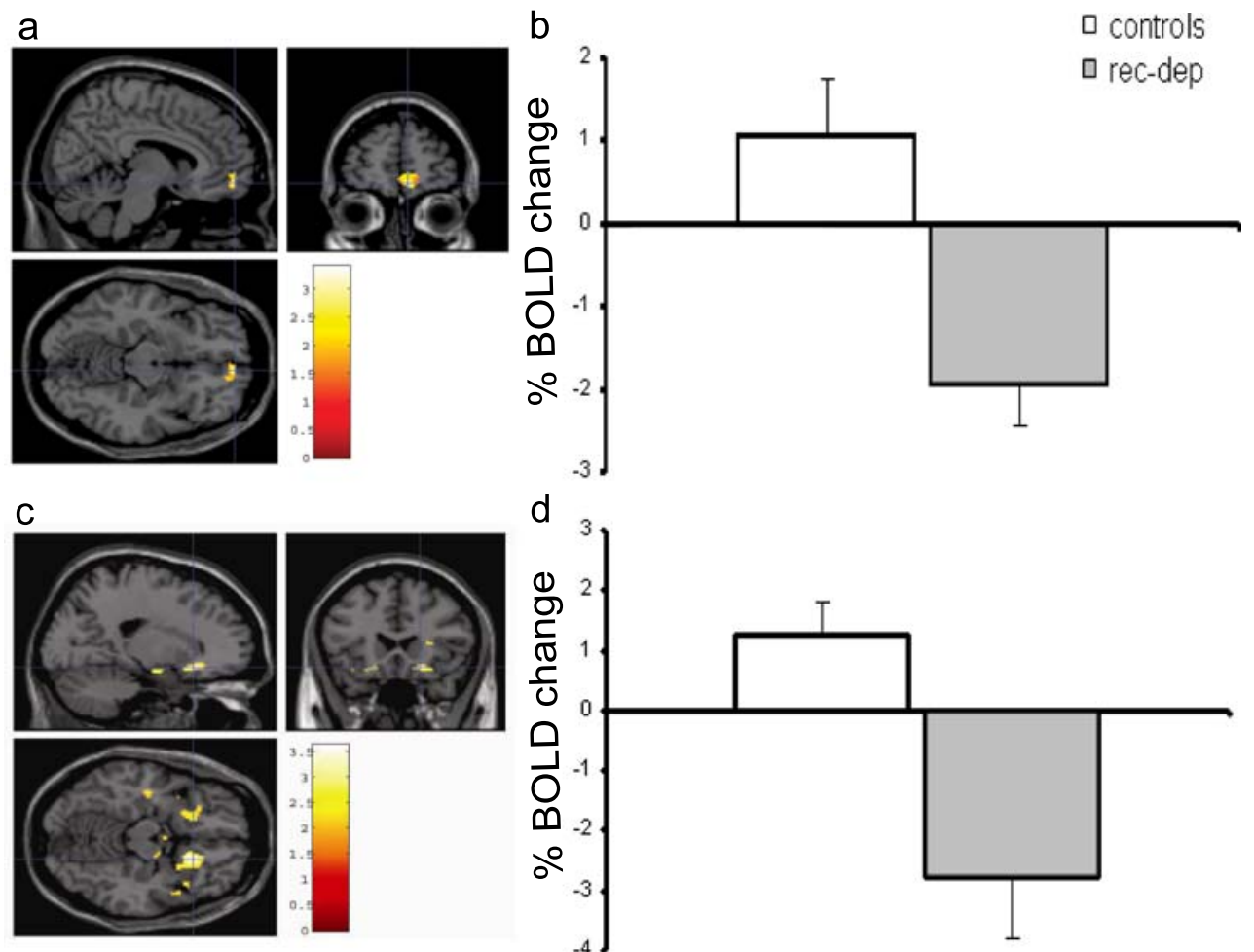
example, Jaspers (1963) noted that “...patients complain that they no longer feel gladness or pain...." Thus, this blunting of response within the lateral orbitofrontal cortex to the combination of the strawberry taste and picture may reflect deficits in the integration of cross-modal sensory input similar to that seen in the VMPFC/medial orbitofrontal cortex. However, future research is required to assess to what extent this supralinearity effect occurs because of reduced impact of multimodal stimuli presentations vs. exaggerated responses to aversive pictorial stimuli.

Consistent with this, the recovered depressed patients also showed increased responses in the caudate nucleus to the aversive sight of the moldy strawberries. This is of interest because the emotion of disgust has been shown to activate the caudate (Phillips et al. 1998; Shapira et al. 2003; Fitzgerald et al. 2004) and this suggests that the recovered depressed patients, in this brain region, show increased processing of aversive cues, consistent with negative biases reported across paradigms in depression (Bradley et al. 1997; Murphy et al. 1999; Surguladze et al. 2004). Such a pattern of effect with increased responses in subcortical areas to aversive stimuli but flattened responses within the prefrontal cortex when cross-modal information is combined in recovered depressed patients provides a compelling parallel to reports of increased negative bias yet often subjective experiences of emotional blunting. This seemingly paradoxical state could arise through increased drive and responses to aversive or ambiguous stimuli at a fairly automatic level coupled with a failure to integrate this fully into subjective experience with multimodal stimuli presentations. At a more practical level, the increased BOLD response at the level of the caudate also suggests that the current results cannot be explained by nonspecific decreases in neural responsivity or neural coupling, but rather that the differences in responses might underlie a vulnerability to experience anhedonia and, therefore, depression.

In conclusion, our findings suggest that patients with a history of depression have impaired neural responses to a primary rewarding stimulus, consistent with the proposal that deficient neural reward processes could be part of an endophenotype of depression. However, it is not clear from the present data whether impairments in reward are present prior to onset of clinical illness or may instead represent a "scar" of the illness or its treatment. Studies in people at high risk of depression, before illness onset, will be needed to resolve this issue. It will also be important to determine whether abnormal neural representations of reward correlate with increased risk of depressive relapse and how far the abnormalities may be reversible with specific psychological and pharmacological treatments.

Acknowledgments We would like to acknowledge Mr. Leo McCabe for the photography and Mrs. Lara Waldenmaier for the help with the questionnaire data analysis. This work has been supported by the Medical Research Council grant no. HQRORVO.

Conflicts of interest Dr Harmer has acted as a consultant for the following companies: Lundbeck, P1Vital, Merck, and Sharpe and Dohme and has received grant income from Merck and Sharpe and Dohme. Professor Cowen has been a paid member of advisory boards of Eli Lilly, Servier, Wyeth, and Xytis and has been a paid lecturer for Eli Lilly, Servier, and GlaxoSmithKline.

Open Access This article is distributed under the terms of the Creative Commons Attribution Noncommercial License which permits any noncommercial use, distribution, and reproduction in any medium, provided the original author(s) and source are credited.

\section{References}

Beck AT, Ward CH, Mendelson M, Mock J, Erbaugh J (1961) An inventory for measuring depression. Arch Gen Psychiatry 4: $561-571$

Blood AJ, Zatorre RJ (2001) Intensely pleasurable responses to music correlate with activity in brain regions implicated in reward and emotion. Proc Natl Acad Sci U S A 98:11818-11823

Bradley BP, Mogg K, Lee SC (1997) Attentional biases for negative information in induced and naturally occurring dysphoria. Behav Res Ther 35:911-927

Cardinal RN, Parkinson JA, Hall J, Everitt BJ (2002) Emotion and motivation: the role of the amygdala, ventral striatum, and prefrontal cortex: a review. Neurosci Biobehav Rev 26:321-352

Collins DL, Neelin P, Peters TM, Evans AC (1994) Automatic 3D intersubject registration of MR volumetric data in standardized Talairach space. J Comput Assist Tomogr 18:192-205

Dryman A, Eaton WW (1991) Affective symptoms associated with the onset of major depression in the community: findings from the US National Institute of Mental Health Epidemiologic Catchment Area Program. Acta Psychiatr Scand 84:1-5

Elliott R, Dolan RJ, Frith CD (2000) Dissociable functions in the medial and lateral orbitofrontal cortex: evidence from human neuroimaging studies. Cereb Cortex 10:308-317

Epstein J, Pan H, Kocsis JH, Yang Y, Butler T, Chusid J et al (2006) Lack of ventral striatal response to positive stimuli in depressed versus normal subjects. Am J Psychiatry 163:1784-1790

Fawcett J, Clark DC, Scheftner WA, Gibbons RD (1983) Assessing anhedonia in psychiatric patients: the Pleasure Scale. Arch Gen Psychiatry 40:79-84

Fitzgerald DA, Posse S, Moore GJ, Tancer ME, Nathan PJ, Phan KL (2004) Neural correlates of internally-generated disgust via autobiographical recall: a functional magnetic resonance imaging investigation. Neurosci Lett 370:91-96

Friston KJ, Worsley KJ, Frackowiak RSJ, Mazziotta JC, Evans AC (1994) Assessing the significance of focal activations using their spatial extent. Hum Brain Mapp 1:214-220

Friston KJ, Glaser DE, Henson RN, Rebel S, Phillips C, Ashburner J (2002) Classical and Bayesian inference in neuroimaging: applications. Neuroimage 16:484-512

Hamilton M (1960) A rating scale for depression. J Neurol Neurosurg Psychiatry 23:56-62

Hare TA, O'Doherty J, Camerer CF, Schultz W, Rangel A (2008) Dissociating the role of the orbitofrontal cortex and the striatum 
in the computation of goal values and prediction errors. J Neurosci 28:5623-5630

Hasler G, Drevets WC, Manji HK, Charney DS (2004) Discovering endophenotypes for major depression. Neuropsychopharmacology 29:1765-1781

Jaspers K (1963) Allgemeine psychopathologie, 5th edn. Springer, Berlin General psychopathology. Manchester University Press, Manchester. Translated by J. Hoenig and M. W. Hamilton

Keedwell PA, Andrew C, Williams SC, Brammer MJ, Phillips ML (2005) The neural correlates of anhedonia in major depressive disorder. Biol Psychiatry 1:843-853

Kiebel SJ, Poline JB, Friston KJ, Holmes AP, Worsley KJ (1999) Robust smoothness estimation in statistical parametric maps using standardized residuals from the general linear model. Neuroimage 10:756-766

Knutson B, Bhanji JP, Cooney RE, Atlas LY, Gotlib IH (2008) Neural responses to monetary incentives in major depression. Biol Psychiatry 63:686-692

Kringelbach ML, Rolls ET (2004) The functional neuroanatomy of the human orbitofrontal cortex: evidence from neuroimagaing and neuropsychology. Prog Neurobiol 72:341-372

Lane RD, Reiman EM, Ahern GL, Schwartz GE, Davidson RJ (1997) Neuroanatomical correlates of happiness, sadness, and disgust. Am J Psychiatry 154:926-933

Maldjian JA, Laurienti PJ, Burdette JB, Kraft RA (2003) An automated method for neuroanatomic and cytoarchitectonic atlas-based interrogation of fMRI data sets. Neuroimage 19:1233-1239

McCabe C, Rolls ET (2007) Umami: a delicious flavour formed by convergence of taste and olfactory pathways in the human brain. Eur J Neurosci 25:1855-1864

McCabe C, Rolls ET, Bilderbeck A, McGlone F (2008) Cognitive influences on the affective representation of touch and the sight of touch in the human brain. Soc Cogn Affect Neurosci 3:97108. doi:10.1093/scan/nsn1005

McClure SM, York MK, Montague PR (2004) The neural substrates of reward processing in humans: the modern role of FMRI. Neuroscientist 10:260-268

Murphy FC, Sahakian BJ, Rubinsztein JS, Michale A, Rogers RD, Robbins TW et al (1999) Emotional bias and inhibitory control processes in mania and depression. Psychol Med 29:1307-1321

Nestler EJ, Carlezon WA Jr (2006) The mesolimbic dopamine reward circuit in depression. Biol Psychiatry 59:1151-1159

O'Doherty J, Kringelbach ML, Rolls ET, Hornak J, Andrews C (2001a) Abstract reward and punishment representations in the human orbitofrontal cortex. Nat Neurosci 4:95-102
O'Doherty J, Rolls ET, Francis S, Bowtell R, McGlone F (2001b) The representation of pleasant and aversive taste in the human brain. $\mathrm{J}$ Neurophysiol 85:1315-1321

Passingham RE, Toni I, Rushworth MF (2000) Specialisation within the prefrontal cortex: the ventral prefrontal cortex and associative learning. Exp Brain Res 133:103-113

Phillips ML, Young AW, Scott SK, Calder AJ, Andrew C, Giampietro $V$ et al (1998) Neural responses to facial and vocal expressions of disgust. Proc Biol Sci 265:1809-1817

Robbins TW, Everitt BJ (1996) Neurobehavioural mechanisms of reward and motivation: a review. Curr Opin Neurobiol 6:228-236

Rolls ET, McCabe C (2007) Enhanced affective brain representations of chocolate in cravers vs. non-cravers. Eur J Neurosci 26: 1067-1076

Rolls ET, Kringelbach ML, de Araujo IET (2003) Different representations of pleasant and unpleasant odours in the human brain. Eur J Neurosci 18:695-703

Rushworth MF, Behrens TE, Rudebeck PH, Walton ME (2007) Contrasting roles for cingulate and orbitofrontal cortex in decisions and social behaviour. Trends Cogn Sci 11:168-176

Schrader GD (1997) Does anhedonia correlate with depression severity in chronic depression? Compr Psychiatry 38:260-263

Shapira NA, Liu Y, He AG, Bradley MM, Lessig MC, James GA et al (2003) Brain activation by disgust-inducing pictures in obsessive-compulsive disorder. Biol Psychiatry 54:751-756

Snaith RP, Hamilton M, Morley S, Humayan A (1995) A scale for the assessment of the hedonic tone: the Snaith-Hamilton Pleasure Scale. Br J Psychiatry 167:99-103

Spitzer RL, Williams JB, Gibbon M, First MB (2004) Structured clinical interview for the DSM-IV (SCID-I/P). American Psychiatric Press, Arlington, VA

Steele JD, Kumar P, Ebmeier KP (2007) Blunted response to feedback information in depressive illness. Brain 130:2367-2374

Surguladze SA, Young AW, Senior C, Brebion G, Travis MJ, Phillips ML (2004) Recognition accuracy and response bias to happy and sad facial expressions in patients with major depression. Neuropsychology 18:212-218

Wilson JL, Jenkinson M, Araujo IET, Kringelbach ML, Rolls ET, Jezzard P (2002) Fast, fully automated global and local magnetic field optimisation for fMRI of the human brain. Neuroimage 17:967-976

Worsley KJ, Marrett P, Neelin AC, Friston KJ, Evans AC (1996) A unified statistical approach for determining significant signals in images of cerebral activation. Hum Brain Mapp 4:58-73

Zald DH, Hagen MC, Pardo JV (2002) Neural correlates of tasting concentrated quinine and sugar solutions. J Neurophysiol 87:1068-1075 